\title{
UM NOVO ÍNDICE DE QUALIDADE DAS ÁGUAS PARA PROTEÇÃo DA VIDA AQUÁTICA APLICADO AO RIO ATIBAIA, REGIÃO DE CAMPINAS/PAULÍNIA - SP
}

\author{
Gilberto Silvério da Silva* \\ Universidade Tecnológica Federal do Paraná, Campus de Medianeira, CP 271, 85884-000 Medianeira - PR, Brasil \\ Wilson de F. Jardim \\ Instituto de Química, Universidade Estadual de Campinas, CP 6154, 13084-971 Campinas - SP, Brasil
}

Recebido em 12/4/05; aceito em 14/10/05; publicado na web em 18/4/06

\begin{abstract}
A NEW WATER QUALITY INDEX FOR PROTECTION OF AQUATIC LIFE APPLLIED TO THE ATIBAIA RIVER, REGION OF CAMPINAS/PAULÍNIA CITIES - SÃO PAULO STATE. In this paper a water quality index is developed to subsidize management actions in the Atibaia River for upon protection of aquatic organisms. This index is composed of two measurable environmental parameters normaly, ammonia and dissolved oxygen, the latter representing the contribution of organic matter. Concentrations of these two variables were normalized on a scale from 0 to 100 and translated into statements of quality (excellent, good, regular, bad and very bad). The index was applied to three monitoring points in the Atibaia River and compared to other indices used by the State of São Paulo Environmental Agency (CETESB). The results showed that the degradation in this watershed follows the urban population density. The developed index is more restricted than the other ones routinely used to infer water quality.
\end{abstract}

Keywords: water quality index; Atibaia River; ammonia and dissolved oxygen.

\section{INTRODUÇÃo}

O objetivo de um Índice de Qualidade das Águas (IQA) é comunicar a qualidade de um determinado corpo hídrico aos atores institucionais de uma bacia hidrográfica, sejam eles a população, as prefeituras, os órgãos de controle ambiental, os comitês das bacias hidrográficas, as organizações não-governamentais, entre outros. Desse modo, o índice de qualidade das águas colabora na construção de um sistema de suporte à tomada de decisão em uma bacia hidrográfica ${ }^{1,2}$.

Uma dificuldade na elaboração de um índice de qualidade das águas é sintetizar em um único número (que pode estar relacionado a um estado da qualidade: ótima, boa, regular, ruim e péssima, por ex.) uma realidade complexa, onde inúmeras variáveis ambientais têm influência. Soma-se a isso, o fato que a qualidade da água é uma função direta de seu uso proposto. Portanto, uma definição clara dos objetivos que se desejam alcançar com este índice de qualidade se faz necessária, pois entre os usos da água estão a irrigação, a recreação, o industrial, o abastecimento público, a manutenção da vida aquática, etc.

Um índice de qualidade das águas pode ser projetado para uma situação específica, inserido no contexto de uma bacia hidrográfica, trazendo consigo a diversidade urbano-industrial presente, a prática de uso e ocupação do solo e o padrão de desenvolvimento tecnológico. Ou seja, a elaboração de um índice de qualidade das águas pode ser simplificada ao levar em consideração somente as variáveis ambientais críticas que afetam determinado corpo hídrico em um determinado momento. Essa redução do número de variáveis ambientais, graças à escolha daquelas que realmente afetam a qualidade, facilita a elaboração de um índice de qualidade, tornando também sua aplicação mais econômica e desse modo aumentando sua possibilidade de uso em diversas bacias hidrográficas do país. Outra vantagem ao se trabalhar com um índice que utiliza poucas variáveis

*e-mail: gilberto@md.cefetpr.br ambientais é a minimização de um clássico e indesejável efeito na elaboração de índices de qualidade das águas, conhecido como efeito eclipse. O efeito eclipse resulta do processo de agregar inúmeras variáveis ambientais em um único número, o que pode produzir uma atenuação do impacto negativo de uma das variáveis frente ao comportamento estável das demais. Os índices propostos na literatura têm procurado diminuir a presença desse efeito eclipse usando diferentes formas matemáticas ${ }^{3}$ para se obter o valor final do índice ou, então, estabelecendo diferentes pesos às variáveis que constituem o índice de qualidade ${ }^{4}$.

Outro aspecto que esse estudo procura abordar diz respeito à toxicidade do composto amônia. Normalmente, os corpos aquáticos são encarados como meros fornecedores de água para suprir as inúmeras demandas criadas pela sociedade, sendo que os critérios de qualidade comumente adotados quase nunca levam em consideração a manutenção da vida aquática nestes corpos, mas sim a qualidade desta água para fins de potabilidade. Isto pode criar algumas distorções quando se estipulam valores máximos permitidos para alguns parâmetros de monitoração rotineira, como amônia, por ex., tendo em vista que a grande maioria dos organismos aquáticos é extremamente sensível a este composto, enquanto que para o ser humano o aspecto odor, e não a toxicidade, é o que impõe seu limite para critérios de potabilidade $^{5}$. O impacto ecológico da amônia nas comunidades aquáticas, em peixes e na população de invertebrados bentônicos, por ex., pode se dar em termos de toxicidade crônica com efeitos sobre a capacidade reprodutiva (produção de ovos e sobrevivência larval), o crescimento (comprimento e peso), o comportamento, os tecidos (mudanças patológicas nos tecidos das brânquias, rins e fígados dos peixes) e alterações bioquímicas e fisiológicas ${ }^{6}$.

A amônia apresenta-se em ambientes aquáticos na forma molecular amônia, $\mathrm{NH}_{3}$, conhecida também como amônia nãoionizada, e na forma iônica amônio, $\mathrm{NH}_{4}^{+}$. A forma não-ionizada é muito mais tóxica que a iônica, certamente graças a sua capacidade de difusão através da membrana epitelial dos organismos aquá- 
ticos. Devido à importância da amônia não-ionizada, tornou-se uma convenção na literatura científica expressar a toxicidade da amônia em termos desse composto. Critérios e padrões de qualidade da água seguiram essa convenção. Contudo, há razões para crer que o íon amônio pode contribuir significativamente para a toxicidade da amônia sob determinadas condições ${ }^{7}$. Neste estudo o termo amônia é genérico, utilizado para referir-se às formas $\mathrm{NH}_{3}$ e $\mathrm{NH}_{4}^{+} \mathrm{em}$ água, indistintamente. Quando se fizer necessário especificar as formas, pois a toxicidade é uma função dessa especiação, será adotada a seguinte terminologia: amônia ou amônia total $\left(\mathrm{NH}_{3}+\mathrm{NH}_{4}^{+}\right)$, amônia não-ionizada $\left(\mathrm{NH}_{3}\right)$ e íon amônio $\left(\mathrm{NH}_{4}^{+}\right)$.

Quanto ao oxigênio dissolvido, $\mathrm{OD}$, uma variável ambiental clássica, sua ausência pode afetar significativamente a biota aquática. De um modo geral e simplificado, com base nos critérios de qualidade para oxigênio dissolvido publicados pela Agência de Proteção Ambiental dos Estados Unidos (EPA) ${ }^{8}$, as faixas de concentração de oxigênio dissolvido com as respectivas comunidades aquáticas que podem suportar tais níveis de oxigênio dissolvido são: de 0 a $2 \mathrm{mg} \mathrm{L}^{-1}$ é insuficiente para manter a vida aquática, de 2 a $4 \mathrm{mg} \mathrm{L}^{-1}$ somente poucas espécies de peixes podem sobreviver, de 4 a $7 \mathrm{mg} \mathrm{L}^{-1}$ é aceitável para peixes de águas quentes e de 7 a $11 \mathrm{mg} \mathrm{L}^{-1}$ é ideal para peixes de águas frias. A Resolução Federal CONAMA 357/059, que trata da qualidade dos corpos hídricos superficiais, traz o valor mínimo permitido de $5 \mathrm{mg} \mathrm{L}^{-1}$ para corpos hídricos classe 2, valor que apresenta uma coerência com os padrões de qualidade preconizados pela referida agência ambiental.

A Resolução Federal CONAMA 357/05 estabelece que corpos hídricos classe 2, caso do Rio Atibaia, onde será aplicado o novo índice, devem manter como uma de suas funções ecológicas ${ }^{10}$ o equilíbrio das comunidades aquáticas e em seu artigo 34, parágrafo primeiro, postula que "O efluente não deverá causar ou possuir potencial para causar efeito tóxico aos organismos aquáticos no corpo receptor...". Este instrumento legal sustenta e abre caminho para um índice de qualidade das águas voltado para a proteção das comunidades aquáticas, pois além de explicitar a necessidade da manutenção da vida aquática, alerta para que a manutenção dessa vida seja norteadora do controle de fontes emissoras de poluentes. Portanto, o índice proposto passa também a desempenhar uma função de indicador de desempenho, capaz de verificar a efetividade das medidas gerenciais de controle de emissões pontuais ou difusas em uma bacia hidrográfica.

\section{PARTE EXPERIMENTAL}

\section{O Rio Atibaia}

O Rio Atibaia, na região das cidades de Campinas e Paulínia, Estado de São Paulo, apresenta-se sob o impacto de uma grande carga poluidora proveniente do esgoto doméstico in natura das cidades presentes em sua bacia hidrográfica. Estudos têm mostrado que as presenças de matéria orgânica e amônia são responsáveis em grande parte pela degradação da qualidade destas águas, afetando o equilíbrio das comunidades aquáticas ${ }^{11,12}$. A bacia do Rio Atibaia está localizada na bacia dos Rios Piracicaba, Capivari e Jundiaí, que compõem a quinta unidade de gerenciamento hídrico do Estado de São Paulo. O Rio Atibaia nasce na cidade de Atibaia, avança pelas regiões das cidades de Campinas e Paulínia e tem sua foz na represa de Americana, onde com o Rio Jaguari forma o Rio Piracicaba. Em sua cabeceira é formado pelos rios Atibainha e Cachoeira, ambos represados para abastecer o sistema Cantareira, principal fonte de água para a Região Metropolitana de São Paulo (Figura 1).

Neste estudo foram utilizados dados referentes a três pontos de monitoração ao longo do Rio Atibaia. Estes dados foram retirados

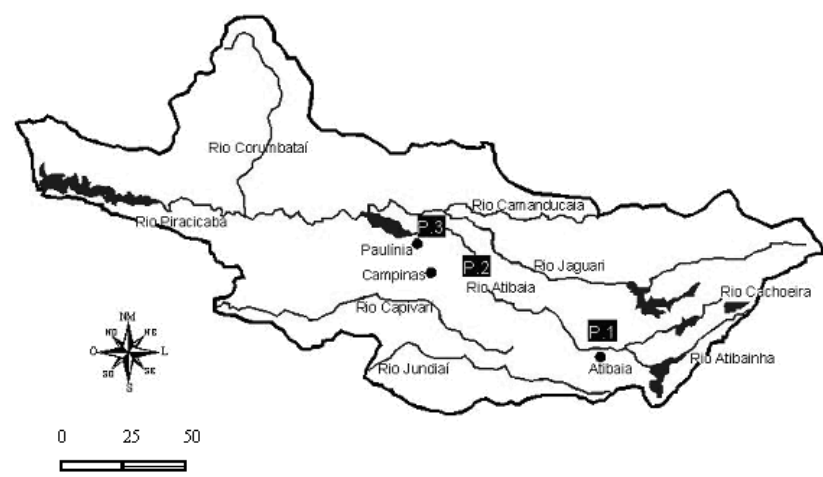

Figura 1. A bacia dos Rios Piracicaba, Capivari e Jundiaí, com destaque para os pontos de monitoração ao longo do Rio Atibaia. Ponto P.1, nascente do Rio Atibaia, na estação de captação de água da cidade de Atibaia. Ponto P.2, localizado na estação de captação de água da cidade de Campinas e Ponto P.3, logo após a afluência do Ribeirão Anhumas, na cidade de Campinas

dos "Relatórios de Qualidade das Águas Interiores do Estado de São Paulo", publicados pela CETESB ${ }^{1}$, referentes aos anos de 2000 a 2003. Para os três pontos mostrados na Figura 1 têm-se os seguintes códigos utilizados pela CETESB: Ponto P.1, código ATIB02010, está localizado na estação de captação de água da cidade de Atibaia, na nascente do Rio Atibaia; Ponto P.2, código ATIB02065, está localizado na estação de captação de água da cidade de Campinas. Nesse ponto, o Rio Atibaia encontra-se sob efeito de considerável carga de esgoto doméstico de inúmeras cidades. O último ponto de monitoração é o Ponto P.3, código ATIB02605, localizado praticamente no final da bacia, estando essas águas sob impacto do Ribeirão Anhumas, responsável pelo aporte de esgoto doméstico in natura de uma população aproximada de 250 mil habitantes da cidade de Campinas ${ }^{2}$.

\section{O desenvolvimento do índice de qualidade das águas para a proteção da vida aquática - IQA $\mathrm{AvA}_{\mathrm{PVA}}$}

Para desenvolvimento do índice de qualidade das águas para a proteção da vida aquática, IQA ${ }_{\mathrm{PVA}}$, considerou-se que as seguintes características deveriam ser atendidas por esse índice: representar a qualidade da água em termos de proteção da vida aquática; contemplar o estado atual de degradação do Rio Atibaia; evitar o efeito eclipse; usar o conceito de operador mínimo; estar focado nas variáveis ambientais críticas que comprometem a qualidade da água; permitir a troca/incorporação das variáveis ambientais críticas de forma simples; facilitar a monitoração; servir de indicador de desempenho na aplicação de controle de fontes pontuais e difusas; dar a mesma importância ambiental às variáveis críticas que o compõem e, incorporar as formas tóxicas dos compostos com, $^{3,411,13-22}$.

Para atender a esses requisitos, o índice proposto foi ${ }^{19,23}$

$\mathrm{IQA}_{\mathrm{PVA}}=$ Min (Amônia total ${ }_{\mathrm{n}}, \mathrm{OD}_{\mathrm{n}}$ )

onde, IQA $\mathrm{PVA}_{\mathrm{PA}}$ é o índice de qualidade das águas para proteção da vida aquática; Amônia total ${ }_{n}$ é a concentração normalizada da amônia total e $\mathrm{OD}_{\mathrm{n}}$ é a concentração normalizada do oxigênio dissolvido, representando um dos impactos ambientais da matéria orgânica nos ecossistemas aquáticos.

A Equação 1 estabelece que o valor numérico do $\mathrm{IQA}_{\mathrm{PVA}}$ é o menor valor normalizado das variáveis ambientais amônia total e oxigênio dissolvido. Smith ${ }^{19}$ e Flores ${ }^{23}$ citam essa mesma forma de calcular o valor final do índice, cujo objetivo é evitar o efeito 
eclipse pelo uso do operador mínimo. A idéia é utilizar a variável ambiental mais degradada, operador mínimo, em termos de proteção da vida aquática para se obter o valor final do índice, similar ao conceito de nutriente-limitante utilizado no estudo da eutrofização, onde um componente pode definir o estado das águas. $\mathrm{O}$ uso do operador mínimo, portanto, constitui-se em uma forma simples de eliminar o clássico efeito eclipse presente na elaboração dos índices de qualidade.

O processo de normalização, que consiste em relacionar os valores numéricos das concentrações das variáveis ambientais da Equação 1 em uma escala de 0 a 100, com o valor 100 representando a melhor qualidade ambiental, pode ser melhor visualizado por intermédio da Tabela 1. Tomando como exemplo os valores das concentrações da amônia total e do oxigênio dissolvido presentes na Tabela 2, referentes à monitoração feita no Rio Atibaia, ponto P.1, no mês de janeiro de 2000, 0,17 $\mathrm{mg} \mathrm{N} \mathrm{L}^{-1}$ e 3,6 $\mathrm{mg} \mathrm{L}^{-1}$, respectivamente, é possível correlacioná-los com as faixas das curvas de normalização mostradas na Tabela 1. A variável amônia total, com sua concentração de $0,17 \mathrm{mg} \mathrm{N} \mathrm{L}^{-1}$, recebe um valor de normalização de 70 . O oxigênio dissolvido, por sua vez, na concentração de $3,6 \mathrm{mg} \mathrm{L}^{-1}$, recebe um valor de normalização de 40. Esses valores de normalização são obtidos com o uso das curvas de Coneza, citadas por Pesce e Wunderlin ${ }^{24}$. Aqui cabe a ressalva da necessidade de se estabelecer curvas de normalização que contemplem, de modo mais apropriado, a realidade ecotoxicológica do Rio Atibaia, considerando-se fatores como $\mathrm{pH}$, temperatura e espécies nativas. Estudos mais recentes sobre a toxicidade crônica da amônia em ambientes aquáticos, publicados pela $\mathrm{EPA}^{7}$, passam a defini-la em função do $\mathrm{pH}$ e da temperatura. Essa ecotoxicidade da amônia adotada pela $\mathrm{EPA}^{7}$ foi incorporada pela Resolução Federal CONAMA 357/059 , sendo que para a classe 2 os valores limites vão de $3,7 \mathrm{mg} \mathrm{N} \mathrm{L}^{-1}$ para $\mathrm{pH}$ igual ou menor que 7,5 até $0,5 \mathrm{mg} \mathrm{N} \mathrm{L}^{-1}$ para $\mathrm{pH}$ acima de 8,5. Todavia, os critérios de qualidade estabelecidos pela $\mathrm{EPA}^{7}$ são constantemente reformulados, sempre que os novos estudos justifiquem uma revisão. Atualmente, a EPA ${ }^{25}$ mostra a intenção de reavaliar os critérios de qualidade para a amônia, destacando estudos com bivalves de água doce, em que algumas espécies mostraram maior sensibilidade a esse poluente.

Ainda na Tabela 1, de posse dos valores normalizados da amônia total e oxigênio dissolvido, 70 e 40, respectivamente, é feita uma relação desses valores com os estados da qualidade (ótima, boa, regular, ruim e péssima). Para se efetuar esta correlação foram utilizadas as faixas de qualidade da $\mathrm{CETESB}^{1}$. As faixas se dividem em ótima para $79<\mathrm{IQA}_{\mathrm{PVA}}<100$, boa para $51<\mathrm{IQA}_{\mathrm{PVA}} \leq 79$, regular para $36<\mathrm{IQA}_{\mathrm{PVA}} \leq 51$, ruim para $19<\mathrm{IQA}_{\mathrm{PVA}} \leq 36$ e péssima para IQA $\leq 19$. É claro que essa divisão proposta tem sua parcela de subjetividade e é susceptível de adequação. Um método de aperfeiçoamento tanto da curva de normalização como das faixas de qualidade da água é a utilização de um painel de especialistas com diferentes bases científicas sobre a qualidade da água, denominado de Método Delphi ${ }^{19}$.

Outra observação com respeito à Equação 1 é sobre o uso de pesos nas variáveis ambientais. Índices clássicos de qualidade das águas costumam ponderar as variáveis ambientais ${ }^{3}$. Essas ponderações geralmente se apresentam na forma de expoentes aos quais as variáveis ambientais normalizadas são elevadas e assumem valores de 0 a 1 . Os valores desses expoentes estão relacionados diretamente à importância ambiental que essas variáveis têm na composição do índice. A Equação proposta para cálculo do índice IQA $\mathrm{PVA}_{\mathrm{PV}}$, Equação 1, não utiliza esse conceito de importância ambiental, uma vez que a escolha do valor mínimo normalizado dispensa seu uso. A expressão proposta na Equação 1 ainda permite que novas variáveis ambientais sejam incorporadas ao índice de qualidade ou que ocorra a substituição das suas variáveis, pois partindo-se do entendimento que a poluição é um processo dinâmico, pode ocorrer que uma nova variável ambiental se mostre crítica a partir de determinado momento. A presença do fenol, por ex., pode ser percebida como um risco para as comunidades aquáticas. A sua incorporação é possível, uma vez que este possua característica ecotoxicológica, aqui compreendida como efeito direto de um composto químico específico sobre as espécies vivas, e apresente uma curva de normalização definida. Ou então, que investimentos em estação de tratamento de esgoto minimizem significativamente a presença da amônia nos corpos hídricos, e esse composto deixe de ser crítico, não justificando mais sua presença no IQA $\mathrm{PVA}_{\mathrm{P}}$. Por fim, pode ser observado que o IQA $\mathrm{PVA}_{\mathrm{P} A}$ proposto utiliza os compostos responsáveis diretamente pelo efeito tóxico, amônia total e oxigênio dissolvido, e não o composto na forma agregada, nitrogênio total, por ex., pois desse modo se estaria incorrendo, por outro caminho, no clássico efeito eclipse.

\section{RESULTADOS E DISCUSSÃO}

$\mathrm{O} \mathrm{IQA}_{\mathrm{PVA}}$ foi aplicado para três pontos ao longo do Rio Atibaia, P.1, P.2 e P.3 (Figura 1) para dados referentes aos anos de 2000 a 2003. As Tabelas 2 a 5 apresentam os estados da qualidade obtidos com o IQA $\mathrm{PVA}_{\text {. }}$. Apresentam também dois índices de qualidade das águas utilizados pela CETESB ${ }^{1}$ e publicados em seus "Relatórios de Qualidade das Águas Interiores do Estado de São Paulo”. O primeiro desses índices é o Índice de Qualidade das Águas (IQA), índice pioneiro no país, lançado no ano de 1975 , voltado à qualidade da água para fins de abastecimento público. A partir de 2002, a CETESB ${ }^{1}$ passou a publicar em seus relatórios um segundo índice, Índice de Qualidade das Águas para Proteção da Vida Aquática (IVA) ${ }^{26}$.

Na Tabela 2, para cada ponto de monitoração P.1, P.2 e P.3, a primeira linha traz o valor da amônia total (Amônia total) e na segunda linha, o valor normalizado (Amônia total ${ }_{n}$ ). Na terceira linha são apresentados os dados para oxigênio dissolvido (OD) e na quarta, os seus valores normalizados $\left(\mathrm{OD}_{\mathrm{n}}\right)$. Na quinta linha está o menor valor quando se compara o valor normalizado da amônia total e de oxigênio dissolvido, aplicação do conceito de operador mínimo. A sexta linha apresenta o índice de qualidade $\mathrm{IQA}_{\mathrm{PVA}} \mathrm{em}$ termos de estados da qualidade (ver Tabela 1). Na sétima linha está o índice da CETESB, IQA. As Tabelas 3 a 5 seguem o mesmo arranjo e referem-se, respectivamente, aos anos de 2001 a 2003.

Pelos dados da Tabela 2, os índices IQA ${ }_{\mathrm{PVA}}$ e IQA apresenta-

Tabela 1. Curvas de normalização para amônia total e oxigênio dissolvido, com os respectivos fatores de normalização e estados da qualidade

\begin{tabular}{lcccccccccccc}
\hline Estados da Qualidade & \multicolumn{3}{c}{ Ótima } & \multicolumn{2}{c}{ Boa } & \multicolumn{2}{c}{ Regular } & & \multicolumn{2}{c}{ Ruim } & Péssima \\
\hline Fator de Normalização & 100 & 90 & 80 & 70 & 60 & 50 & 40 & 30 & 20 & 10 & 0 \\
Amônia total(mg N L & -1 & $<0,01$ & $<0,05$ & $<0,10$ & $<0,20$ & $<0,30$ & $<0,40$ & $<0,50$ & $<0,75$ & $<1,00$ & $\leq 1,25$ & $>1,25$ \\
Oxigênio dissolvido (mg L & $\geq 7,5$ & $>7,0$ & $>6,5$ & $>6,0$ & $>5,0$ & $>4,0$ & $>3,5$ & $>3,0$ & $>2,0$ & $\geq 1,0$ & $<1,0$ \\
\hline
\end{tabular}

As concentrações da amônia total e do oxigênio dissolvido, juntamente com o fator de normalização, foram retiradas das curvas de Coneza, citadas por Pesce e Wunderlin ${ }^{24}$. Os estados da qualidade foram estabelecidos com base nas faixas usadas pela CETESB ${ }^{1}$ para seu IQA: Ótima para $79<\mathrm{IQA}_{\mathrm{PVA}}<100$, Boa para $51<\mathrm{IQA}_{\mathrm{PVA}} \leq 79$, Regular para $36<\mathrm{IQA}_{\mathrm{PVA}} \leq 51$, Ruim para $19<\mathrm{IQA}_{\mathrm{PVA}} \leq 36$ e Péssima para IQA $\leq 19$. 
ram um comportamento similar no que se refere a estados da qualidade. Eles apontam para estados mais degradados à medida que se avança na bacia hidrográfica do Rio Atibaia, fato esperado, pois o aporte de amônia e matéria orgânica vai se intensificando ao longo da bacia. Contudo, ocorre um distanciamento entre esses dois índices, isto é, os estados da qualidade retratados pelo IQA $\mathrm{PVA}_{\mathrm{P}}$ mostram-se mais degradados em relação àqueles mostrados pelo IQA quando se aproxima da foz do Rio Atibaia. A Tabela 3, que apresenta os dados para o ano de 2001, mostra resultados que seguem o mesmo comportamento visto para os dados da Tabela 2.

Para os anos de 2002 e 2003, Tabelas 4 e 5, respectivamente, surge nos relatórios da $\mathrm{CETESB}^{1}$ o Índice de Qualidade para Pro-

Tabela 2. Comparação entre os índices de qualidade IQA ${ }_{\mathrm{PVA}}$ e IQA para o ano de 2000

\begin{tabular}{|c|c|c|c|c|c|c|c|c|}
\hline & & & Jan & Mar & Mai & Jul & Set & Nov \\
\hline \multirow[t]{21}{*}{2000} & \multirow[t]{7}{*}{ P.1 } & Amônia total $\left(\mathrm{mg} \mathrm{N} \mathrm{L}^{-1}\right)$ & 0,17 & 0,03 & 0,13 & 0,26 & 0,08 & 0,018 \\
\hline & & Amônia total & 70 & 90 & 70 & 60 & 80 & 70 \\
\hline & & $\mathrm{OD}\left(\mathrm{mg} \mathrm{L}^{-1}\right)$ & 3,6 & 5,7 & 6,1 & 5,7 & 5 & 4,7 \\
\hline & & $\mathrm{OD}_{\mathrm{n}}$ & 40 & 60 & 70 & 60 & 50 & 50 \\
\hline & & Operador Mínimo & 40 & 60 & 70 & 60 & 50 & 50 \\
\hline & & $\mathrm{IQA}_{\mathrm{PVA}}$ & Regular & Boa & Boa & Boa & Regular & Regular \\
\hline & & IQA & Boa & Boa & Boa & Boa & Regular & Boa \\
\hline & \multirow[t]{7}{*}{ P.2 } & Amônia total (mg N $\left.{ }^{\mathrm{L}-1}\right)$ & 0,49 & 0,47 & 0,47 & 1,8 & 0,61 & 0,89 \\
\hline & & Amônia total & 40 & 40 & 40 & 0 & 30 & 20 \\
\hline & & $\mathrm{OD}\left(\mathrm{mg} \mathrm{L}^{-1}\right)$ & 6,2 & 6,7 & 7 & 5 & 5,5 & 6,4 \\
\hline & & $\mathrm{OD}_{\mathrm{n}}$ & 70 & 80 & 80 & 50 & 60 & 70 \\
\hline & & Operador Mínimo & 40 & 40 & 40 & 0 & 30 & 20 \\
\hline & & $\mathrm{IQA}_{\mathrm{PVA}}$ & Regular & Regular & Regular & Péssima & Ruim & Ruim \\
\hline & & IQA & Regular & Boa & Regular & Regular & Boa & Regular \\
\hline & \multirow[t]{7}{*}{ P.3 } & Amônia total $\left(\mathrm{mg} \mathrm{N} \mathrm{L}^{-1}\right)$ & 0,63 & 1,27 & 2,5 & 4,6 & 1,2 & 1 \\
\hline & & Amônia total & 30 & 0 & 0 & 0 & 10 & 10 \\
\hline & & $\mathrm{OD}\left(\mathrm{mg} \mathrm{L} \mathrm{L}^{-1}\right)$ & 5,4 & 5,9 & 5,5 & 5,6 & 5,7 & 6 \\
\hline & & $\mathrm{OD}_{\mathrm{n}}$ & 60 & 60 & 60 & 60 & 60 & 60 \\
\hline & & Operador Mínimo & 30 & 0 & 0 & 0 & 10 & 10 \\
\hline & & $\mathrm{IQA}_{\mathrm{PVA}}$ & Ruim & Péssima & Péssima & Péssima & Péssima & Péssima \\
\hline & & IQA & Ruim & Regular & Regular & Regular & Regular & Regular \\
\hline
\end{tabular}

Os valores de amônia total, oxigênio dissolvido (OD) e IQA foram retirados do relatório de águas interiores publicados pela CETESB ${ }^{1}$. O valor de $\mathrm{IQA}_{\mathrm{PVA}}$ foi calculado segundo a Equação 1 e os estados da qualidade foram baseados nos valores da CETESB para seu IQA. Os valores normalizados da Amônia total (Amônia total $\left.{ }_{n}\right)$ e $O D\left(D_{n}\right)$ foram calculados segundo a Tabela 1.

Tabela 3. Comparação entre os índices de qualidade IQA ${ }_{\mathrm{PVA}}$ e IQA para o ano de 2001

\begin{tabular}{|c|c|c|c|c|c|c|c|}
\hline & & Jan & Mar & Mai & Jun & Set & Nov \\
\hline \multirow[t]{7}{*}{ P.1 } & Amônia total (mg N L-1) & 0,60 & 0,46 & 0,37 & 0,10 & 0,27 & 0,05 \\
\hline & Amônia total & 30 & 50 & 60 & 80 & 70 & 100 \\
\hline & $\mathrm{OD}\left(\mathrm{mg} \mathrm{L}^{-1}\right)^{\mathrm{n}}$ & 4,6 & 6,0 & 5,8 & 6,3 & 4,9 & 4,5 \\
\hline & $\mathrm{OD}_{\mathrm{n}}$ & 50 & 70 & 60 & 70 & 50 & 50 \\
\hline & Operador Mínimo & 30 & 50 & 60 & 70 & 50 & 50 \\
\hline & $\mathrm{IQA}_{\mathrm{PVA}}$ & Ruim & Regular & Boa & Boa & Regular & Regular \\
\hline & IQA $^{\text {PVA }}$ & Regular & Boa & Boa & Boa & Boa & Boa \\
\hline \multirow[t]{7}{*}{ P. 2} & Amônia total (mg N L-1) & 0,39 & 0,63 & 0,85 & 1,3 & 1,2 & 1,6 \\
\hline & Amônia total & 50 & 30 & 20 & 0 & 10 & 0 \\
\hline & $\mathrm{OD}\left(\mathrm{mg} \mathrm{L}^{-1}\right)^{\mathrm{n}}$ & 7,0 & 6,2 & 6,7 & 6,4 & 4,6 & 4,6 \\
\hline & $\mathrm{OD}_{\mathrm{n}}$ & 80 & 70 & 80 & 70 & 50 & 50 \\
\hline & Operador Mínimo & 50 & 30 & 20 & 0 & 10 & 0 \\
\hline & $\mathrm{IQA}_{\mathrm{PVA}}$ & Regular & Ruim & Ruim & Péssima & Péssimo & Péssimo \\
\hline & IQA & Boa & Regular & Boa & Boa & Regular & Regular \\
\hline \multirow[t]{7}{*}{ P.3 } & Amônia total (mg N L-1) & 1,07 & 1,1 & 1,9 & 3,2 & 0,8 & 2,4 \\
\hline & Amônia total & 10 & 10 & 0 & 0 & 20 & 0 \\
\hline & $\mathrm{OD}\left(\mathrm{mg} \mathrm{L}^{-1}\right)^{\mathrm{n}}$ & 6,0 & 5,2 & 5,8 & 5,3 & 4,8 & 4,7 \\
\hline & $\mathrm{OD}_{\mathrm{n}}$ & 60 & 60 & 60 & 60 & 50 & 50 \\
\hline & Operador Mínimo & 10 & 10 & 0 & 0 & 20 & 0 \\
\hline & $\mathrm{IQA}_{\mathrm{PVA}}$ & Péssimo & Péssima & Péssima & Ruim & Péssima & Péssima \\
\hline & IQA $^{\text {PVA }}$ & Regular & Regular & Regular & Boa & Regular & Regular \\
\hline
\end{tabular}

Aplicam-se as considerações feitas no rodapé da Tabela 2. 
Tabela 4. Comparação entre os índices de qualidade IQA ${ }_{\mathrm{PVA}}$, IVA e IQA para o ano de 2002

\begin{tabular}{|c|c|c|c|c|c|c|c|}
\hline & & Jan & Mar & Mai & Jun & Set & Nov \\
\hline \multirow[t]{8}{*}{ P. 1} & Amônia total $\left(\mathrm{mg} \mathrm{N} \mathrm{L}^{-1}\right)$ & 0,23 & 0,05 & 0,2 & 0,05 & 1,5 & 0,1 \\
\hline & Amônia total & 60 & 80 & 60 & 80 & 0 & 70 \\
\hline & $\mathrm{OD}\left(\mathrm{mg} \mathrm{L}^{-1}\right)$ & 4,4 & 4,6 & 5,6 & 6,5 & 4,8 & 4,2 \\
\hline & $\mathrm{OD}_{\mathrm{n}}$ & 50 & 50 & 60 & 70 & 50 & 50 \\
\hline & Operador Mínimo & 50 & 50 & 60 & 70 & 0 & 50 \\
\hline & $\mathrm{IQA}_{\mathrm{PVA}}$ & Regular & Regular & Boa & Boa & Péssima & Regular \\
\hline & IVA & Regular & Regular & Boa & Ruim & & \\
\hline & IQA & Boa & Regular & Boa & Boa & & \\
\hline \multirow[t]{8}{*}{ P. 2} & Amônia total $\left(\mathrm{mg} \mathrm{N} \mathrm{L}^{-1}\right)$ & 0,74 & 0,35 & 0,5 & 1,1 & 0,58 & 0,69 \\
\hline & Amônia total ${ }_{n}$ & 30 & 50 & 30 & 10 & 30 & 30 \\
\hline & $\mathrm{OD}\left(\mathrm{mg} \mathrm{L}^{-1}\right)$ & 5,9 & 6,1 & 6,8 & 5,9 & 6,9 & 4,5 \\
\hline & $\mathrm{OD}_{\mathrm{n}}$ & 60 & 70 & 80 & 60 & 80 & 50 \\
\hline & Operador Mínimo & 30 & 50 & 30 & 10 & 30 & 30 \\
\hline & $\mathrm{IQA}_{\mathrm{PVA}}$ & Ruim & Regular & Ruim & Péssima & Ruim & Ruim \\
\hline & IVA & Regular & Regular & Boa & Regular & Boa & Ruim \\
\hline & IQA & Regular & Regular & Boa & Regular & Regular & Regular \\
\hline \multirow[t]{8}{*}{ P.3 } & Amônia total $\left(\mathrm{mg} \mathrm{N} \mathrm{L}^{-1}\right)$ & 0,58 & 0,52 & 1,7 & 1,6 & 1,5 & 2,6 \\
\hline & Amônia total ${ }_{n}$ & 30 & 30 & 0 & 0 & 0 & 0 \\
\hline & $\mathrm{OD}\left(\mathrm{mg} \mathrm{L}^{-1}\right)^{\mathrm{n}}$ & 5,6 & 6 & 4,4 & 4,7 & 4,8 & 4,6 \\
\hline & $\mathrm{OD}_{\mathrm{n}}$ & 60 & 60 & 50 & 50 & 50 & 50 \\
\hline & Operador Mínimo & 30 & 30 & 0 & 0 & 0 & 0 \\
\hline & $\mathrm{IQA}_{\mathrm{PVA}}$ & Ruim & Ruim & Péssima & Péssima & Péssima & Péssima \\
\hline & IVA & Regular & Ruim & Ruim & Ruim & Ruim & Ruim \\
\hline & IQA & Воа & Regular & Regular & Regular & Regular & Regular \\
\hline
\end{tabular}

Aplicam-se as considerações feitas no rodapé da Tabela 2, acrescentando o Índice Para Proteção da Vida Aquática (IVA ${ }^{26}$ ) lançado no ano de 2002 pela CETESB $^{1}$.

Tabela 5. Comparação entre os índices de qualidade IQA ${ }_{\mathrm{PVA}}$, IVA e IQA para o ano de 2003

\begin{tabular}{|c|c|c|c|c|c|c|c|c|}
\hline & & & Jan & Mar & Mai & Jun & Set & Nov \\
\hline \multirow[t]{24}{*}{2003} & \multirow[t]{8}{*}{ P. 1} & Amônia total(mg N L $\left.{ }^{-1}\right)$ & 0,27 & 0,76 & 0,26 & 0,27 & 0,05 & 0,2 \\
\hline & & Amônia total & 60 & 20 & 60 & 60 & 80 & 60 \\
\hline & & $\mathrm{OD}\left(\mathrm{mg} \mathrm{L}^{-1}\right)^{\mathrm{n}}$ & 4,2 & 4,8 & 5,8 & 6,6 & 6,4 & 5 \\
\hline & & $\mathrm{OD}_{\mathrm{n}}$ & 50 & 50 & 60 & 80 & 70 & 50 \\
\hline & & Operador Mínimo & 50 & 20 & 60 & 60 & 70 & 50 \\
\hline & & $\mathrm{IQA}_{\mathrm{PVA}}$ & Regular & Ruim & Boa & Boa & Boa & Regular \\
\hline & & IVA & Regular & Ruim & Boa & Boa & Regular & Regular \\
\hline & & IQA & Regular & Boa & Boa & Boa & Boa & Boa \\
\hline & \multirow[t]{8}{*}{ P.2 } & Amônia total $\left(\mathrm{mg} \mathrm{N} \mathrm{L}^{-1}\right)$ & 0,24 & 0,78 & 1 & 0,8 & 0,53 & 0,91 \\
\hline & & Amônia total & 60 & 20 & 10 & 20 & 30 & 20 \\
\hline & & $\mathrm{OD}\left(\mathrm{mg} \mathrm{L}^{-1}\right)^{\mathrm{n}}$ & 5,8 & 6,3 & 6,4 & 7,5 & 4,8 & 5,2 \\
\hline & & $\mathrm{OD}_{\mathrm{n}}$ & 60 & 70 & 70 & 100 & 50 & 60 \\
\hline & & Operador Mínimo & 60 & 20 & 10 & 20 & 30 & 20 \\
\hline & & $\mathrm{IQA}_{\mathrm{PVA}}$ & Boa & Ruim & Péssima & Ruim & Ruim & Ruim \\
\hline & & IVA $_{\text {CETESB }}$ & Regular & Regular & Regular & Regular & Regular & Regular \\
\hline & & $\mathrm{IQA}_{\mathrm{CETESB}}$ & Regular & Regular & Regular & Regular & Regular & Regular \\
\hline & \multirow[t]{8}{*}{ P.3 } & Amônia total $\left(\mathrm{mg} \mathrm{N} \mathrm{L}^{-1}\right)$ & 0,19 & 1,1 & 2,4 & 1,5 & 4,6 & 2,1 \\
\hline & & Amônia total & 70 & 10 & 0 & 0 & 0 & 0 \\
\hline & & $\mathrm{OD}\left(\mathrm{mg} \mathrm{L}^{-1}\right)^{\mathrm{n}}$ & 5,2 & 5,4 & 4,8 & 6,2 & 6,2 & 5,4 \\
\hline & & $\mathrm{OD}_{\mathrm{n}}$ & 60 & 60 & 50 & 70 & 70 & 60 \\
\hline & & Operador Mínimo & 60 & 10 & 0 & 0 & 0 & 0 \\
\hline & & $\mathrm{IQA}_{\mathrm{PVA}}$ & Boa & Péssima & Péssima & Péssima & Péssima & Péssima \\
\hline & & IVA & Ruim & Ruim & Ruim & Ruim & Ruim & Ruim \\
\hline & & IQA & Regular & Regular & Boa & Regular & Regular & Regular \\
\hline
\end{tabular}

Aplicam-se as considerações feitas no rodapé da Tabela 4. 
teção da Vida Aquática ${ }^{26}$, IVA. Os dados referentes a esses anos mostram a tendência observada para os anos de 2000 e 2001, ou seja, maior deterioração dos estados da qualidade à medida que se avança para a foz do Rio Atibaia. Os resultados dos índices também mostram uma hierarquia no incremento dessa degradação. O IQA $\mathrm{PVA}_{\mathrm{P}}$ apresenta uma degradação maior que o IVA que, por sua vez, apresenta uma degradação maior que o IQA. A maior degradação da qualidade da água mostrada pelo IQA ${ }_{\mathrm{PVA}}$ pode ter sua justificativa na ausência do efeito eclipse e pelas variáveis que o compõem, permitindo com isso uma resposta mais sensível desse índice à forte presença do esgoto doméstico in natura, rico em amônia e matéria orgânica. Essa degradação apontada pelo $\mathrm{IQA}_{\mathrm{PVA}}$ sinaliza aos tomadores de decisão da bacia do Rio Atibaia sobre a importância de investimentos em tratamento de esgoto, e focaliza melhor o papel poluidor do esgoto doméstico in natura na bacia do Rio Atibaia quando comparado com a forte presença industrial pois, com exceção do ponto de monitoração P.1, o operador mínimo utilizado foi o valor normalizado da amônia total, cuja fonte predominante é urbana, de origem doméstica ${ }^{11}$.

\section{CONCLUSÕES}

$\mathrm{O}$ índice proposto neste trabalho, $\mathrm{IQA}_{\mathrm{PVA}}$, mostrou-se capaz de comunicar a qualidade das águas do Rio Atibaia quando em comparação com os índices utilizados pela CETESB $^{1}$ (IQA e IVA), revelando, contudo, uma capacidade de refletir de modo mais acentuado a presença poluidora do esgoto doméstico na bacia do Rio Atibaia. Os estados da qualidade do $\mathrm{IQA}_{\mathrm{PVA}}$ mostraram uma situação crítica para o Rio Atibaia em termos de capacidade de manutenção da vida aquática, exigência preconizada para corpos hídricos classe 2, servindo assim para o subsídio de ações gerenciais e sinalizando para o papel nada desprezível do esgoto urbano doméstico in natura no histórico de poluição dessa bacia, anos de 2000 a 2003, apesar da forte presença industrial ali existente. O IQA ${ }_{\mathrm{PVA}}$, utilizando apenas duas variáveis ambientais facilmente mensuráveis, amônia total e oxigênio dissolvido, pode ser útil no gerenciamento de bacias hidrográficas onde o esgoto doméstico in natura é preocupante, situação essa comum em nosso país que apresenta baixíssimos índices de tratamento de esgoto. O índice encontra ainda aplicação em outros cenários, como por ex., em bacias hidrográficas onde o uso e a ocupação do solo apresentam características agropecuárias, com atividades de suinocultura e avicultura, ou onde a presença industrial emissora desses poluentes esteja presente, como em fábricas de fertilizantes, refinarias de petróleo e agroindústrias. O IQA ${ }_{\mathrm{PVA}}$ pode ser ainda utilizado na avaliação de desempenho de medidas para controle de fontes pontuais e difusas em bacias hidrográficas. Por esses aspectos, o IQA ${ }_{\mathrm{PVA}}$ se apresenta como mais uma ferramenta disponível no sistema de gestão de bacias hidrográficas e pode ser conectado de diferentes formas na estrutura de monitoração de um corpo hídrico, seja pela sua implantação, devido a custos, em bacias com ausência de um programa estabelecido de monitoração, seja de forma complementar a outros índices por questões de economia ou necessidade, seja em conjunto com índices bióticos.

\section{AGRADECIMENTOS}

À UNICAMP, FAPESP, CAPES, CETESB e UTFPR. Agradecemos também aos assessores de Química Nova pelas sugestões dadas à forma final deste texto.

\section{REFERÊNCIAS}

1. Companhia de Tecnologia de Saneamento Ambiental (CETESB); Relatório de Qualidade das Águas Interiores do Estado de São Paulo, São Paulo, 2000 a 2003.

2. Companhia de Tecnologia de Saneamento Ambiental (CETESB); Watershed Management 2000 - Brasil/Canadá, São Paulo, 2000.

3. Landwehr, J. M.; Deininger, R. A.; Journal WPCF 1976, 48, 954.

4. House, M. A.; Ellis, J. B.; Water Sci. Technol. 1987, 19, 145.

5. Ministério da Saúde; Portaria $n^{o} .518$ de 25 de março de 2004 Brasília, DF, 2004.

6. Environmental Canada; Priority Substances List - Assessment Report Ammonia in The Aquatica Environment - May 2000, Canadá, 2000.

7. Environmental Protection Agency of United State (USEPA); 1999 Update of ambient water quality criteria for ammonia - EPA 822-R-99-014, Washington, 1999.

8. Environmental Protection Agency of United State (USEPA); Ambient Water Quality Criteria for Dissolved Oxygen - EPA 440586003, Washington, DC, 1986.

9. Conselho Nacional de Meio Ambiente (CONAMA); Resolução $n^{\circ} .357$ de 15 de março de 2005. Brasília, DF, 2005.

10. Ministério do Meio Ambiente; Gerenciamento de Bacias Hidrográficas, Brasília, DF, 1995.

11. Silva, G. S.; Tese de Doutorado, Universidade Estadual de Campinas, Brasil, 2004.

12. Comitê das Bacias Hídricas dos Rios Piracicaba, Capivari e Jundiaí; Relatório Zero, Piracicaba, 2000.

13. Prati, L.; Pavalello, R.; Pesarin, F.; Water Res. 1971, 5, 741.

14. Walski, T. M.; Parker, F. L.; J. Environ. Eng. Div. 1974, 593.

15. Inhaber, H.; Water Res. 1975, 9, 821.

16. Ross, S. L.; Water Pollut. Control 1977, 113.

17. Tyson, J. M.; House, M. A.; Water Sci. Technol. 1989, 21, 1149.

18. Smith, D. G.; Water Sci. Technol. 1989, 21, 123.

19. Smith, D. G.; Water Res. 1990, 24, 1237.

20. Novotny, V.; Water Sci. Technol. 1996, 33, 1.

21. Stambuk-Giljanovic, N.; Water Res. 1999, 33, 3423.

22. Bordalo, A.A; Nilsumranchit, W. ; Chalermwat, K.; Water Res. 2001, 35, 3635.

23. Flores, J. C.; Water Res. 2002, 36, 4664.

24. Pesce, S. F.; Wunderlin, D. A.; Water Res. 2000, 34, 2915.

25. http://www.epa.gov/waterscience/criteria/ammonia/re-eval-fs.htm, acessada em Julho 2005.

26. Zagatto, P. A.; Lorenzetti, M. L.; Lamparelli, M. C.; Salvador, M. E. P.; Menegon J. R.; Acta Limnol. Bras. 1999, 11, 111. 